\title{
Un estudio fonético-acústico preliminar sobre las consonantes nasales sordas de la lengua resígaro (arawak)
}

\section{A preliminary phonetic-acoustic study on the voiceless nasal consonants of the Resígaro (Arawak) language}

\author{
Jhon Jimenez Peña \\ Universidad Nacional Mayor de San Marcos, Lima Perú \\ jhon.jimenez@unmsm.edu.pe \\ https://orcid.org/oooo-0003-3317-6152
}

\begin{abstract}
Resumen
Este artículo representa una primera descripción acústica de las consonantes nasales sordas de la lengua resígaro (arawak). Se exploran algunas características como la estructura acústica, la frecuencia y la intensidad del pico más prominente y la duración de esta clase consonantes. Las particularidades encontradas fueron comparadas con las de otras lenguas que presentan estos sonidos con el fin de encontrar semejanzas y diferencias como también catalogarlas preliminarmente en algún grupo tipológico. Además, presentamos la metodología empleada para el recojo de datos, el análisis de los datos y las conclusiones.
\end{abstract}

Palabras clave: consonantes nasales sordas, resígaro, arawak, fonética acústica, lenguas amazónicas

\begin{abstract}
This paper provides a first acoustic description of the nasal voiceless consonants of the Resígaro (Arawak) language. Some features such as acoustic structure, frequency and intensity of the most prominent peak and duration of this consonant class are explored. The particularities found were compared with those of other languages that present these sounds in order to find similarities and differences, as well as to catalog them preliminarily in some typological group. In addition, the methodology used for data collection, data analysis and conclusions are presented.
\end{abstract}

Keywords: voiceless nasal consonants, Resígaro, Arawak, acoustic phonetics, Amazonian languages

\section{Resumo}

Este artigo representa uma primeira descrição acústica das consoantes nasais sem voz da língua Resígaro (Arawak). Algumas características como a estrutura acústica, frequência e intensidade do pico mais proeminente e duração desta classe consonante são exploradas. As particularidades encontradas foram comparadas com as de outras línguas que apresentam estes sons, a fim de encontrar semelhanças e diferenças, bem como de as catalogar preliminarmente em algum grupo tipológico. Além disso, apresentamos a metodologia utilizada para a recolha de dados, análise de dados e conclusões.

Palavras-chave: consoantes nasais sem voz, Resígaro, Arawak, fonética acústica, línguas amazónicas 
Un estudio fonético-acústico preliminar sobre las consonantes nasales sordas de la... Jhon Jimenez Peña

\section{Introducción}

La lengua resígaro (Iso 639-3, rgr) pertenece a la familia lingüística arawak (Allin, 1976; Payne, 1985). Los resígaros habitaban, anteriormente, territorios entre los ríos Caquetá y Putumayo; sin embargo, producto de la migración a causa de la época del caucho y enfermedades, los hablantes del resígaro se asentaron en territorio peruano en la cuenca del río Ampiyacu, Mariscal Ramón Castilla, región Loreto (Allin, 1976, p. 1).

Actualmente, según el Documento Nacional de Lenguas Originarias del Perú (MINEDU, 2018, p. 241) es una lengua en proceso de extinguirse, pues, cuenta con escasos hablantes que viven en la comunidad de Nueva Esperanza, ubicada a orillas del río Ampiyacu (Loreto), Distrito de Pebas, provincia de Mariscal Ramón Castilla, región Loreto. Pese a que se informaba que solo habría dos hablantes del resígaro (Fagua, 2015), según un estudio meticuloso que realizó el Ministerio de Educación del Perú en el año 2017, esta lengua cuenta con 8 hablantes (MINEDU, 2017).

Los estudios gramaticales del resígaro son escasos como la gran mayoría de lenguas originarias peruanas. Esta lengua cuenta con una gramática escrita por Trevor Allin en el año 1976. En cuanto los estudios fonético-acústicos en esta lengua, son nulos actualmente; sin embargo, se está elaborando una tesis titulada «Análisis acústico de las vocales del resígaro» por la lingüista sanmarquina María Giuliana Tito Quinteros. Por tanto, la presente investigación representa la primera descripción instrumental (acústica) sobre un grupo específico de sonidos en la lengua resígaro.

El resígaro es una lengua aglutinante y tonal. En cuanto su inventario fonológico, esta lengua presenta un amplio número de consonantes que asciende a treinta y posee diez vocales entre cortas y largas. A continuación, se presenta en el Tabla 1 y Tabla 2 las consonantes y vocales del resígaro respectivamente:

Tabla 1. Consonantes del resígaro

\begin{tabular}{l|l|l|l|l|l|l|}
\multicolumn{2}{c|}{} & Labial & Alveolar & Palatal & Velar & Glotal \\
\hline \multirow{2}{*}{ Oclusivas } & Aspirada & $\mathrm{p}^{\mathrm{h}}$ & $\mathrm{t}^{\mathrm{h}}$ & & $\mathrm{k}^{\mathrm{h}}$ & \\
\cline { 2 - 8 } & Sorda & $\mathrm{p}$ & $\mathrm{t}$ & $\mathrm{t}^{\mathrm{j}}$ & $\mathrm{k}$ & $\mathrm{l}$ \\
\cline { 2 - 7 } & Sonora & $\mathrm{b}$ & $\mathrm{d}$ & $\mathrm{d}^{\mathrm{j}}$ & $\mathrm{g}$ & \\
\hline
\end{tabular}


Un estudio fonético-acústico preliminar sobre las consonantes nasales sordas de la...

Jhon Jimenez Peña

\begin{tabular}{|c|c|c|c|c|c|c|}
\hline & & & & & \\
\hline & & Labial & Alveolar & Palatal & Velar & Glotal \\
\hline \multirow{3}{*}{ Africadas } & Aspirada & & $\mathrm{ts}^{\mathrm{h}}$ & $\widehat{t}^{\mathrm{h}}$ & & \\
\hline & Sorda & & ts & $\widehat{t} \int$ & & \\
\hline & Sonora & & $\mathrm{dz}$ & d3 & & \\
\hline \multirow{2}{*}{ Fricativa } & Sorda & $\phi$ & s & $c ̧$ & & $\mathrm{~h}$ \\
\hline & sonora & $\beta$ & & j & & \\
\hline \multirow{2}{*}{ Nasales } & Sorda & $\mathrm{m}_{\mathrm{o}}$ & n̊ & $\mathrm{n}_{0}$ & & \\
\hline & Sonora & $\mathrm{m}$ & $\mathrm{n}$ & $\mathrm{n}$ & & \\
\hline
\end{tabular}

Nota. Adaptado de «A grammar of Resígaro» (Trevor, 1976, p. 47).

Tabla 2. Vocales del resígaro

\begin{tabular}{l|l|l|l} 
& Anterior & Central & Posterior \\
\hline Alta & i i: & & u u: \\
\hline Media & e e: & & o o: \\
\hline baja & & a a: & \\
\hline
\end{tabular}

Nota. Adaptado de «A grammar of Resígaro» (Trevor, 1976, p. 48).

Como se observa en ambos cuadros, esta lengua presenta un sistema fonológico extenso e interesante que necesita una documentación acústica urgentemente. Ahora bien, dentro del grupo de las consonantes, se evidencia una clase de consonantes raras y poca estudiadas en las lenguas del mundo. El resígaro posee una serie consonantes nasales sordas fonológicas $/ \mathrm{m}_{\mathrm{o}} \mathrm{n}_{\mathrm{o}} \mathrm{n}_{\mathrm{o}} /$. Esta clase de consonantes han sido reportadas principalmente en lenguas en el sudeste de asiático (Chirkova, K., Basset, P., y Amelot, A, 2019). En américa del sur, conforme a una búsqueda en el sitio web de Inventarios Fonológicos Suramericanos (sA phon v2.1.o), se registra que hay seis lenguas que presentan consonantes nasales sordas, entre ellas, el resígaro y el baniwa, ambas lenguas de la familia lingüística arawak.

Según Allin (1976), el resígaro posee un contraste entre consonantes nasales sonoras $/ \mathrm{m} /, \mathrm{n} / \mathrm{y} / \mathrm{n} / \mathrm{y}$ sus contrapartes nasales sordos $/ \mathrm{m}_{\mathrm{o}} /, \mathrm{n}_{\mathrm{o}} / \mathrm{y} / \mathrm{j}_{\mathrm{o}} / ;$ sin embargo, como ya se mencionó, la confirmación de esta clase de sonidos se debe una perspectiva impresionista y no ha habido alguna corroboración desde una perspectiva acústica. Por ello, en esta investigación, se tratará de responder la siguiente pregunta: ¿cuáles son las características fonético-acústicas que presentan las 
Un estudio fonético-acústico preliminar sobre las consonantes nasales sordas de la... Jhon Jimenez Peña

consonantes nasales sordas del resígaro? El objetivo de la investigación que guiará la investigación es realizar una descripción exploratoria contrastándola con las investigaciones de Dantsuji (1984, 1986), Bhaskararao y Ladefoged (1991) y Chirkova, K., Basset, P., y Amelot, A. (2019).

\section{Marco referencial}

\subsection{Consonantes nasales $y$ consonantes nasales sordas}

Las consonantes nasales, por su naturaleza, se caracterizan por ser sonantes, es decir, presentan sonoridad espontánea (Chomsky y Halle, 1979). En las lenguas del mundo, las nasales $/ \mathrm{m} / \mathrm{y} / \mathrm{n} /$ son las más recurrentes y las demás aparecen en menor porcentaje (Maddieson, 1984). También existen consonantes nasales, en menor número, con diferente fonación como creaky voice y breathy voice (Ladefoged y Maddieson, 1996; Maddieson, 1984), en las cuales la sonoridad también está involucrada.

Sin embargo, hay un pequeño grupo de lenguas que poseen consonantes nasales sordas (Maddieson, 1984) dentro de su inventario fonológico, las cuales son tipológicamente raras, ya que escapan de la «naturaleza» sonante prototípica de una nasal ya que no son sonantes ni mucho menos sonoras. Por otro lado, la existencia de consonantes nasales sordas en una lengua exige la presencia de sus contrapartes sonoras obligatoriamente (Maddieson, 1984, p. 14).

\subsection{Las consonantes nasales sordas en las lenguas del mundo}

Maddieson (1984, p. 61, 235-239) señala que doce lenguas presentan consonantes nasales sordas de las trecientos siete que poseen consonantes nasales conforme a la base de datos de la UCLA denominada UPSID (Phonological Segment Inventory Database). Cabe resaltar que el resígaro no está incluido en el listado presentado por el autor. Estas lenguas son las siguientes:
a. Sedang
e. Burmese
i. Hopi
b. Lakkia
f. Yao
j. Aleut
c. Sui
g. Klamath
k. Otomi
d. Iai
h. Mazuhua
1. Irish 
Un estudio fonético-acústico preliminar sobre las consonantes nasales sordas de la...

Jhon Jimenez Peña

Ahora bien, existen seis lenguas en américa del sur que poseen consonantes nasales sordas según el sitio web de Inventarios Fonológicos Suramericanos (sAphon v2.1.o) donde se incluye al resígaro; no obstante, Chirkova, K., Basset, P., y Amelot, A. (2019, p. 1) sostienen que las consonantes sordas se encuentran principalmente en lenguas del sudeste asiático y están representadas específicamente por las lenguas tibeto-burmano.

\subsection{Características acústicas de las consonantes nasales sordas}

Dantsuji (1984, p. 5-6) realiza una descripción acústica de las consonantes nasales de la lengua birmano. En este estudio, se presenta la estructura acústica, picos prominentes, intensidad y la duración de esta clase de sonidos. Este autor identifica que las consonantes nasales sordas en birmano están constituidas por dos fases denominadas: (1) porción ruidosa (noisy friction) o fricción nasal (nasal friction). En este fragmento, solo hay fricción, no existe barra de sonoridad ni estriaciones, por lo que se puede sostener que esta parte es sorda; (2) la segunda porción de esta clase de sonidos se le denomina nasal sonora (voiced nasal). Este último fragmento presenta estriciones y sonoridad. Por último, al Dantsuji (1984, p. 7) señala las siguientes generalidades: la intensidad de la parte fricción es más baja que la parte nasal sonora y la duración de la parte de la fricción nasal es mucho mayor a la de la parte nasal sonora.

Bhaskararao y Ladefoged (1991) realizan un estudio acústico sobre consonantes nasales sordas en birmano, mizo y angami. Las conclusiones que se arribaron en este estudio apuntan a una clasificación de esta clase de nasales. Estos autores señalan que las consonantes nasales sordas pueden clasificarse en dos grupos:

Grupo 1: En este grupo se encuentran las consonantes nasales sordas del birmano y el mizo. Estas consonantes se caracterizan porque inician con un período, caracterizado por un flujo de aire (airflow) nasal y oral, y terminan en un período, caracterizado solo por un flujo de aire nasal. La primera parte es sorda (no tiene barra de sonoridad ni estraciones), mientras que la última parte presenta sonoridad (presencia de estriaciones). El flujo de aire nasal continúa por un corto tiempo en la vocal antes de que el velo cierre el conducto (Chirkova, K., Basset, P., y Amelot, A., 2019, p. 4).

Grupo 2: En este grupo se encuentran las consonantes nasales sordas del angami. Estas presentan una estructura acústica diferente en el que las nasales 
Un estudio fonético-acústico preliminar sobre las consonantes nasales sordas de la... Jhon Jimenez Peña

sordas comienzan con un período caracterizado solo por el flujo de aire nasal y terminan en un período caracterizado por el flujo de aire nasal y oral. Estas nasales sordas permanecen sordas a lo largo de la articulación nasal e incluso más allá de la liberación (la vocal puede estar sonora solo parcialmente al principio). El flujo de aire nasal continuo persiste en la siguiente vocal. Este subtipo de nasales sordas también se conoce como «nasales sordas aspiradas» porque se caracterizan por la misma relación de tiempo entre las articulaciones oral y glotal que se observa en las oclusiones aspiradas (Chirkova, K., Basset, P., y Amelot, A., 2019, p. 4).

En resumen, una clase de consonantes nasales sordas se caracterizan por presentar dos fases donde la primera se caracteriza por no presentar sonoridad y la segunda parte sí la presenta. La otra clase se caracteriza por ser completamente sorda, pero el flujo de aire oral y nasal se extiende más de modo que se forma una porción de aspiración; por eso se les conoce «nasales sordas aspiradas». La representación de estos subtipos se puede apreciar en la Figura 1:

Figura 1. Estructura de los dos tipos de nasal sorda

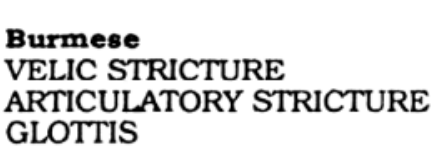

Angami

VELIC STRICTURE ARTICULATORY STRICTURE GLOTTIS
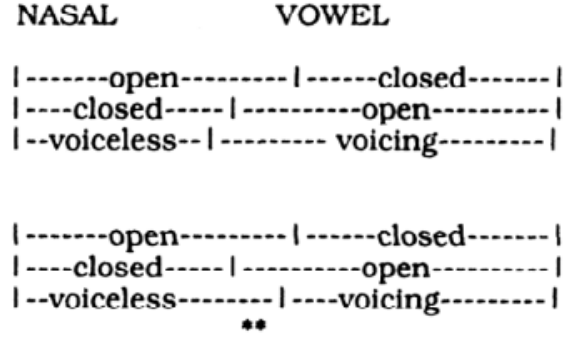

Nota. Representación gráfica de la superposición de la vibración de las cuerdas vocales, apertura del velo del y liberación de la estructura articulatoria de las nasales sordas en birmano y angami. Los símbolos ${ }^{*}$ indican la porción de aspiración. Adaptado de Blankenship, Ladefoged, Bhaskararao y Chase, 1993, p. 78.

\section{Metodología}

Para esta investigación se han usado datos recopilados durante el mes de marzo de 2016, año en el que se realizó un trabajo de campo en la comunidad de Nueva Esperanza, distrito de Pebas, provincia de Mariscal Cáceres, región Loreto para el proceso de normalización del alfabeto ocaina. Durante la estadía, se halló a dos personas hablantes de la lengua resígaro, un varón y una mujer de 67 y 65 años respectivamente, que radicaban en la comunidad de Nueva Esperanza. Ellos 
Un estudio fonético-acústico preliminar sobre las consonantes nasales sordas de la... Jhon Jimenez Peña

compartían una relación de parentesco de hermanos y ambos fueron identificados como pertenecientes de la etnia resígaro por Allin (1976, p. viii). Lamentablemente, en ese mismo año, la hablante resígaro fallece de manera que solo hay un hablante que radica en la comunidad mencionada en la actualidad.

\subsection{Elaboración de los instrumentos}

Principalmente, se crearon listas de palabras objetivo donde contenían a las consonantes nasales sordas. Estas fueron elaboradas a partir de la data documentada en el Vocabulario resígaro (Trevor, 1979) mediante los programas que provee sIL: Toolbox (versión 1.6.2) y Phonology Assistant (versión 3.5.4). En la Figura 2, se muestra la base de datos creada en Toolbox y en la Figura 3 una lista de palabras generada en Phonlogy Assistant.

Figura 2. Interfaz del programa Toolbox (versión 1.6.2)

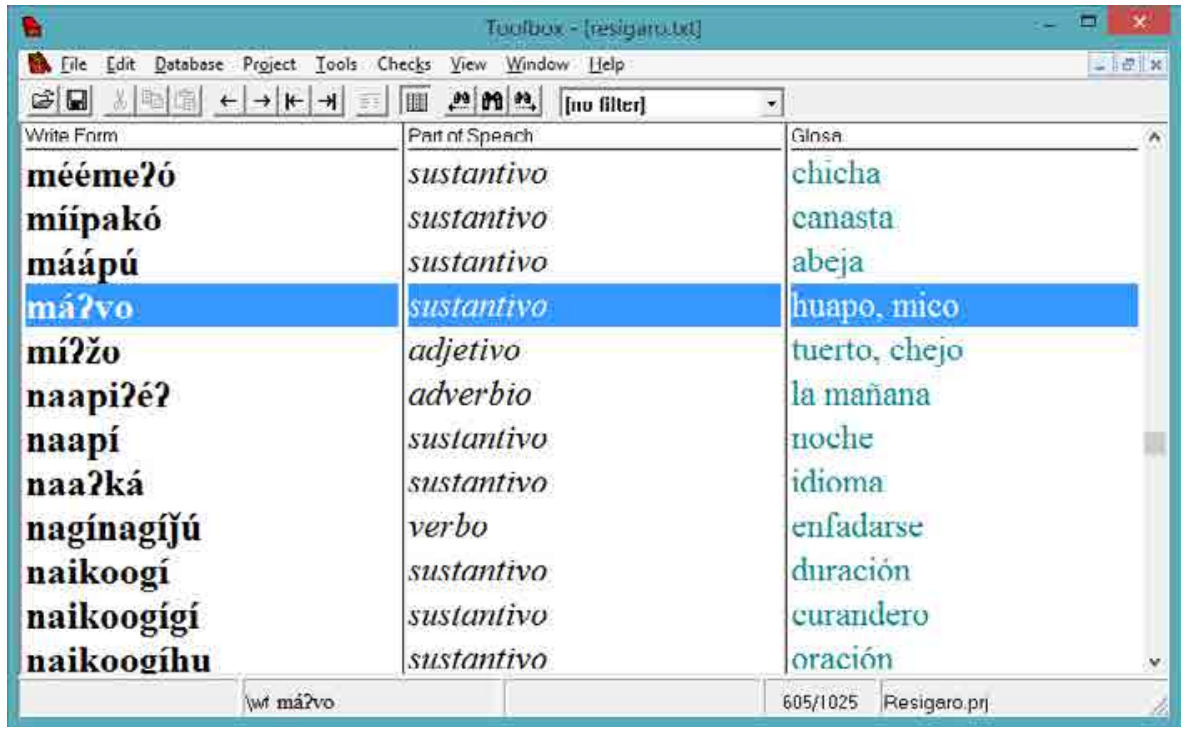

Nota. Base de datos en Toolbox elaborado a partir del Vocabulario resígaro (Trevor, 1979) 
Un estudio fonético-acústico preliminar sobre las consonantes nasales sordas de la... Jhon Jimenez Peña

Figura 3. Interfaz del programa Phonology Assistant (versión 3.5.4)

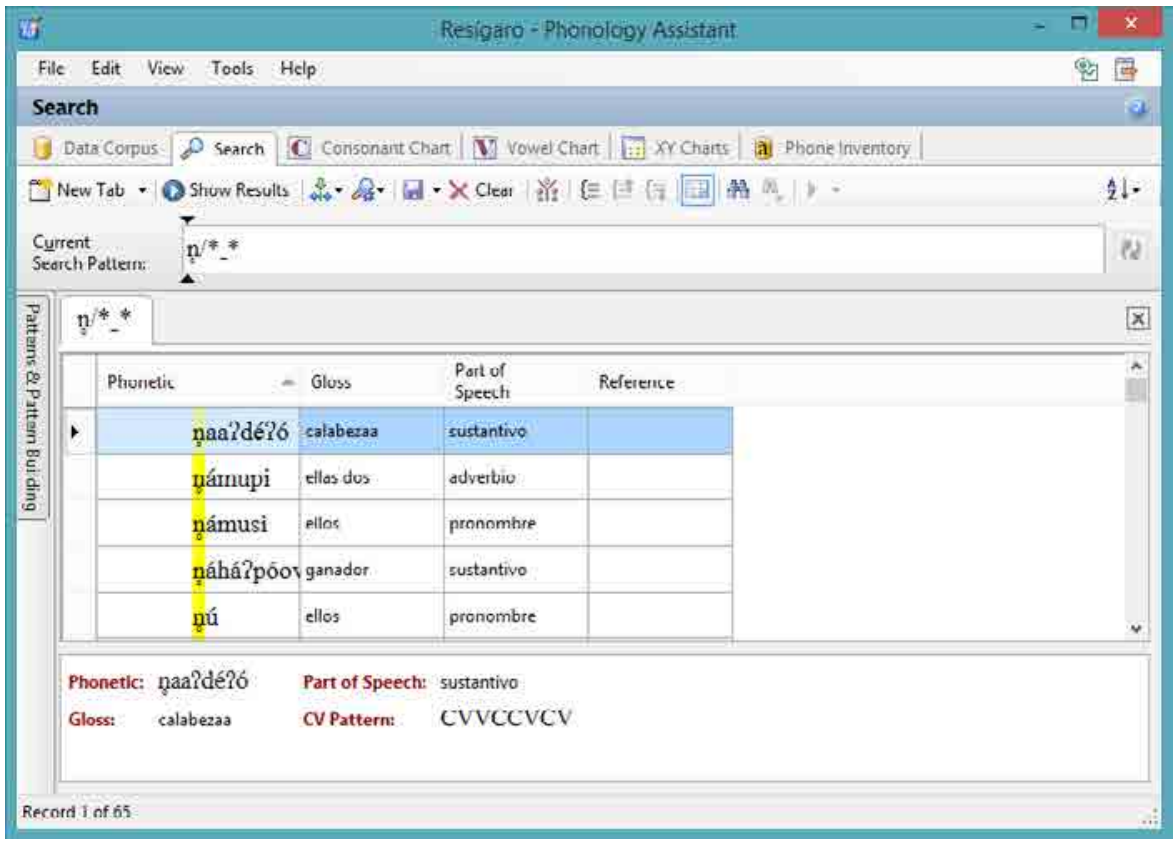

Nota. Selección de palabras que contiene nasales sordas alveolares en Phonology Assistant

\subsection{Recolección de datos}

El protocolo de recojo de datos consistió en la técnica de elicitación. Se siguió los lineamientos que recomiendan Ladefoged (2003) y Bhaskararao y Ladefoged (1991). Los autores recomiendan en introducir la palabra objetivo (se encuentra subrayado) en una oración como «Yo digo la palabra casa en castellano». Para el caso del resígaro, se usó la siguiente oración: [nokem ${ }^{{ }} \dot{t}$ ] 'yo digo la palabra ' (traducción libre).

Se planificó grabar 45 palabras por cada hablante. Estas debían contener las tres consonantes nasales sordas en ataque silábico en posición inicial e intermedio, pero no se pudo identificar adecuadamente la consonante nasal palatal sorda / $/ \mathrm{n} /$ en las palabras donde debería aparecer. Por tal motivo, este estudio preliminar no tomará en cuenta esta consonante. Finalmente, se recopilaron quince palabras que contenían la nasal bilabial sorda $/ \mathrm{m}_{\mathrm{o}} /$ y quince palabras que contenían la nasa alveolar sorda $/ \mathrm{n}_{\mathrm{o}} /$ por cada hablante. Las grabaciones se hicieron en un ambiente sin ruido. 


\subsection{Herramientas de grabación}

Las grabaciones se realizaron con una grabadora Zoom $\mathrm{H}_{4}$ n. Esta nos permitió grabar audios en formato no comprimido WAV, en modo mono, digitalizados con un muestreo de frecuencia de $44100 \mathrm{~Hz}$ y una cuantización lineal de 16 bit. Para Ladefoged (2003), estos parámetros garantizan la obtención de audios en alta calidad para los análisis acústicos.

También usamos un micrófono Shure SM35-XLR, un micrófono condensador cardioide con conector XLR que se sujeta a la cabeza del entrevistado. Este micrófono es unidireccional, es decir, que es sensible a una única dirección y relativamente sordo a cualquier sonido externo. Además, presenta una frecuencia mínima de respuesta de $45 \mathrm{~Hz}$, cifra que se aproxima al promedio de la frecuencia mínima de respuesta de la audición humana que es de $20 \mathrm{~Hz}$.

\subsection{Segmentación, etiquetado} y análisis de los datos

Luego de ordenar los archivos de audios en una computadora personal, se procedió a segmentar los sonidos nasales sordos y etiquetar mediante el programa para el análisis acústico de voz Praat (Versión 5.4.17). Posteriormente, se realizaron los análisis pertinentes con este mismo software consignados en la sección 4 de este artículo.

\section{Análisis y resultados}

\subsection{Análisis a partir de espectrogramas}

Dantsuji (1984) realiza una descripción acústica sobre las consonantes nasales en burmese. En el apartado del análisis llamado constitución, sostiene que esta clase de consonantes presenta una porción de fricción sin sonoridad ni estriaciones. En la revisión de los datos de ambos hablantes, se detectó que las consonantes nasales sordas $\left[\mathrm{m}_{\mathrm{o}}\right]$ y $\left[\mathrm{n}_{\mathrm{o}}\right]$ del resígaro presentan esa porción de fricción sin barra de sonoridad ni estriaciones verticales por lo que podemos sostener que esa fase en resígaro es sorda al igual que el burmese. Esta característica se puede apreciar en la Figura 4 y 5 . Se advierte que no hay indicios de aspiración en la vocal siguiente. 
Un estudio fonético-acústico preliminar sobre las consonantes nasales sordas de la... Jhon Jimenez Peña

Figura 4. Espectrograma de la palabra [mepoko] 'piraña'

TRK1-77

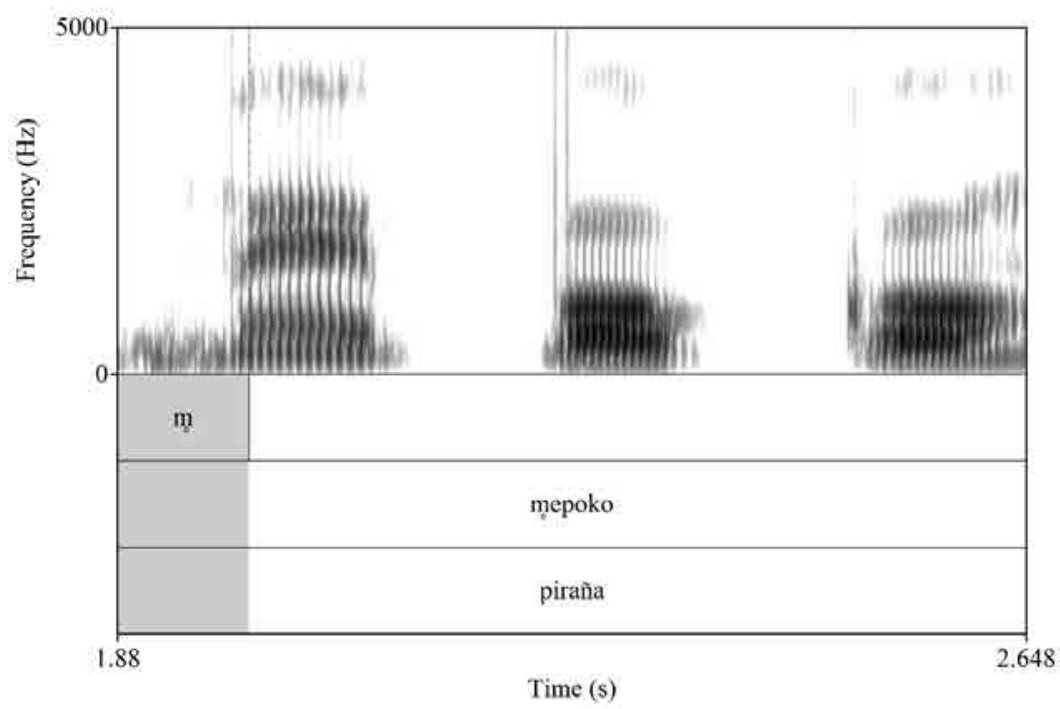

Nota. Se visualiza que el segmento nasal bilabial sordo $[\mathrm{m}]$ no presenta sonoridad. Palabra pronunciada por el hablante varón.

Figura 5. Espectrograma de la frase [no:pa] 'mi pie' mi pic_sc_hinchó

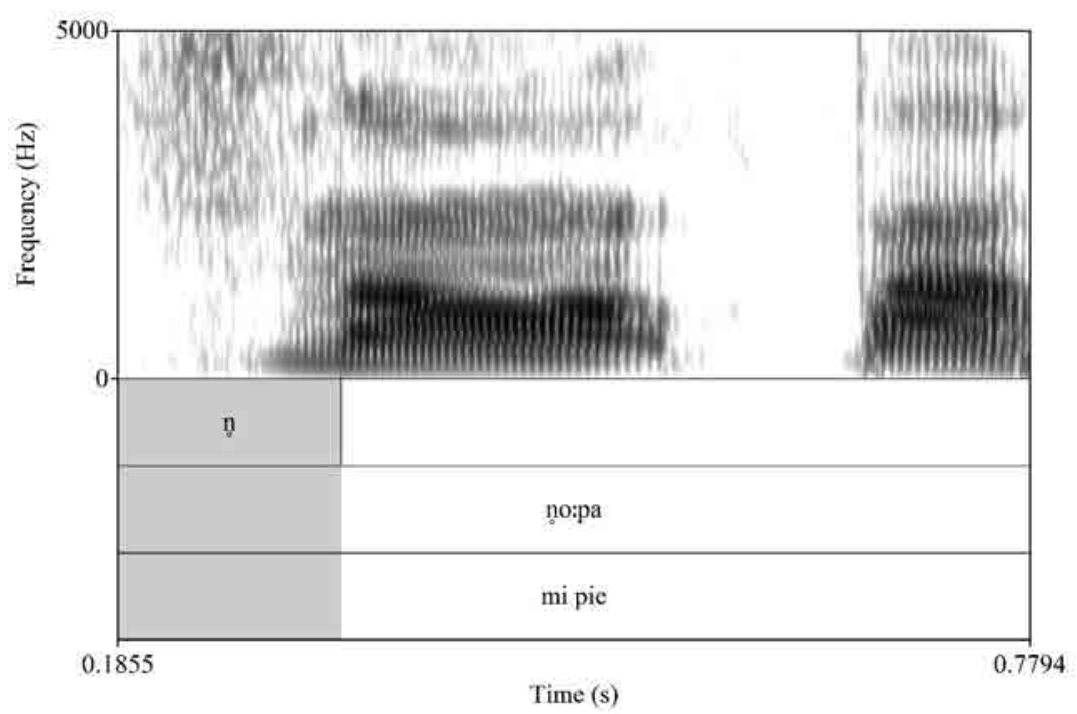

Nota. Se visualiza que el segmento nasal bilabial sordo [n] no presenta sonoridad. Frase pronunciada por la hablante mujer. 
Un estudio fonético-acústico preliminar sobre las consonantes nasales sordas de la...

Jhon Jimenez Peña

Dantsuji (1984) también sostiene que esta clase de sonidos presentan una porción nasal sonora que aparece adyacente a la vocal. En el análisis de los datos, se ha observado que las consonantes nasales sordas del resígaro también presentan esta característica y es el mismo patrón en ambos hablantes.

Para evidenciarlo presentamos espectrogramas de banda estrecha para observar los armónicos, típicos en sonidos sonantes. En la Figura 6 y Figura 7, la porción de nasal sonora de las consonantes nasales aparece en la parte derecha al entrar en contacto con la vocal que le sigue (también está presente en la Figuras 4 y 5). Nótese que en esta porción los armónicos son muy sobresalientes y marcados como ocurre con cualquier sonido sonante. Se advierte que no se han detectado indicios de aspiración en la vocal siguiente en los datos analizados.

Figura 6. Espectrograma de banda estrecha de la palabra [kamiíido] 'uvilla'

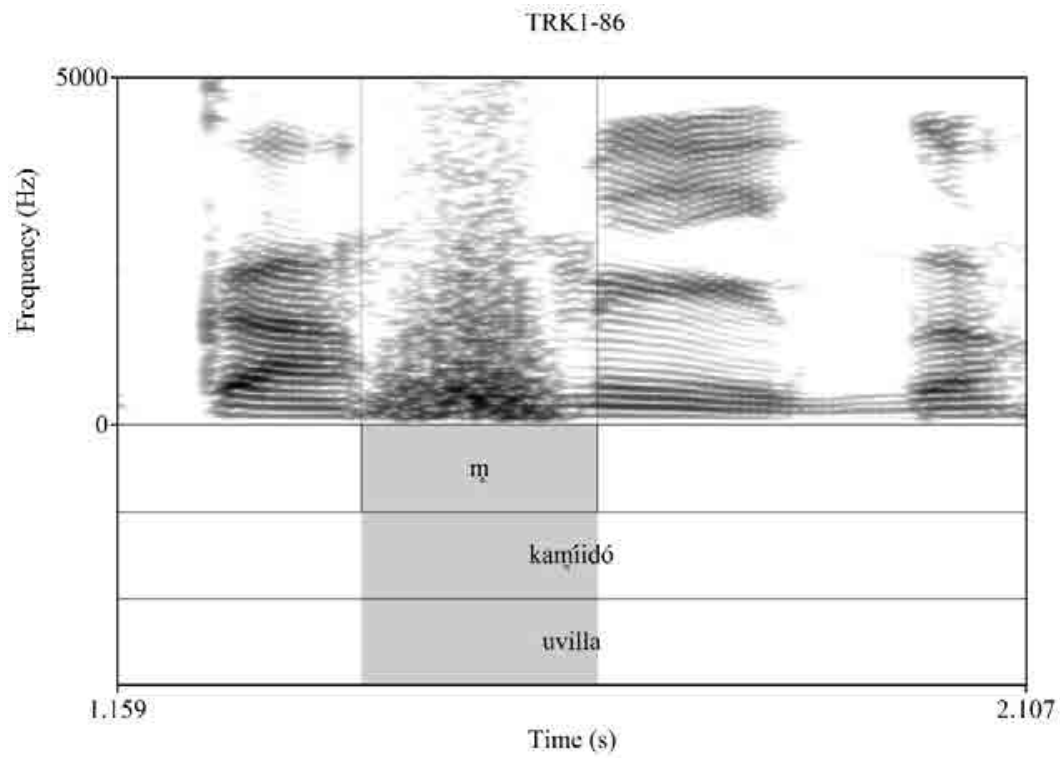

Nota. Se visualiza que la parte final del segmento nasal bilabial sordo [m] existe un fragmento de sonoridad que asumiremos es la porción de nasal sonora. Nótese los armónicos. Palabra pronunciada por el hablante resígaro. 
Un estudio fonético-acústico preliminar sobre las consonantes nasales sordas de la... Jhon Jimenez Peña

Figura 7. Espectrograma de banda estrecha de la palabra [mani ] 'llamar'

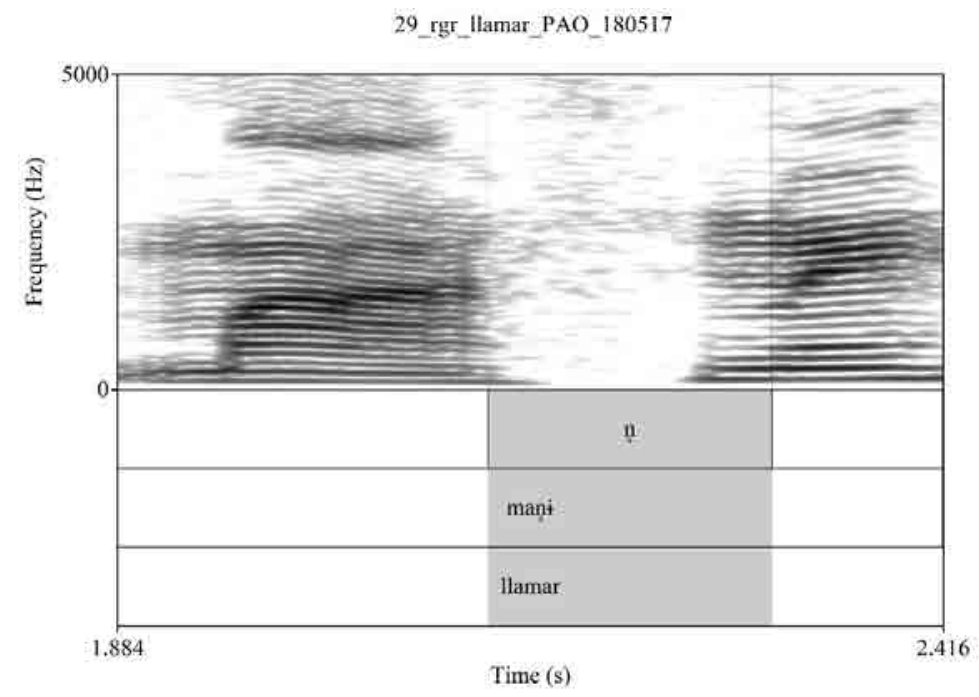

Nota. Se visualiza que la parte final del segmento nasal bilabial sordo [n] existe un fragmento de sonoridad que asumiremos es la porción de nasal sonora. Nótese los armónicos. Palabra pronunciada por la hablante resígaro.

\subsection{Análisis espectral}

Dantsuji (1984) también estudia los picos prominentes de parte de fricción y la intensidad de esta fase y la parte sonora de las consonantes nasales sordas en birmano. Se ha obtenido resultados semejantes al de Dantsuji (1984); sin embargo, esta sección reportará solo información del pico más prominente y la intensidad de $\left[\mathrm{m}_{\mathrm{o}}\right]$ y $\left[\mathrm{n}_{\mathrm{o}}\right]$, parámetro acústico que el autor mencionado no proporciona información dicho autor.

A continuación, se presenta espectros LPC (un gráfico que representa los picos de mayor amplitud) consonantes nasales sordas $\left[m_{0}\right]$ y $\left[n_{0}\right]$ en la Figura 8 y Figura 9 , respectivamente. Cabe resaltar que los valores que se presentan no son promedios, sino solo valores referenciales y representativos de todos los datos analizados. Se requiere más datos para ofrecer promedios. La frecuencia pico más prominente de una consonante nasal bilabial sorda [ $\mathrm{m}_{\mathrm{o}}$ ] se ubica por los $2590 \mathrm{~Hz}$ y tienen una intensidad de $20 \mathrm{~dB}$. Por otra parte, el pico más prominente de la consonante nasal alveolar $\left[\mathrm{n}_{\mathrm{o}}\right]$ se encuentra por los $4629 \mathrm{~Hz}$ y con una intensidad de $17.7 \mathrm{~dB}$. 
Un estudio fonético-acústico preliminar sobre las consonantes nasales sordas de la... Jhon Jimenez Peña

Figura 8. Espectro LPC de una consonante nasal sorda [m]

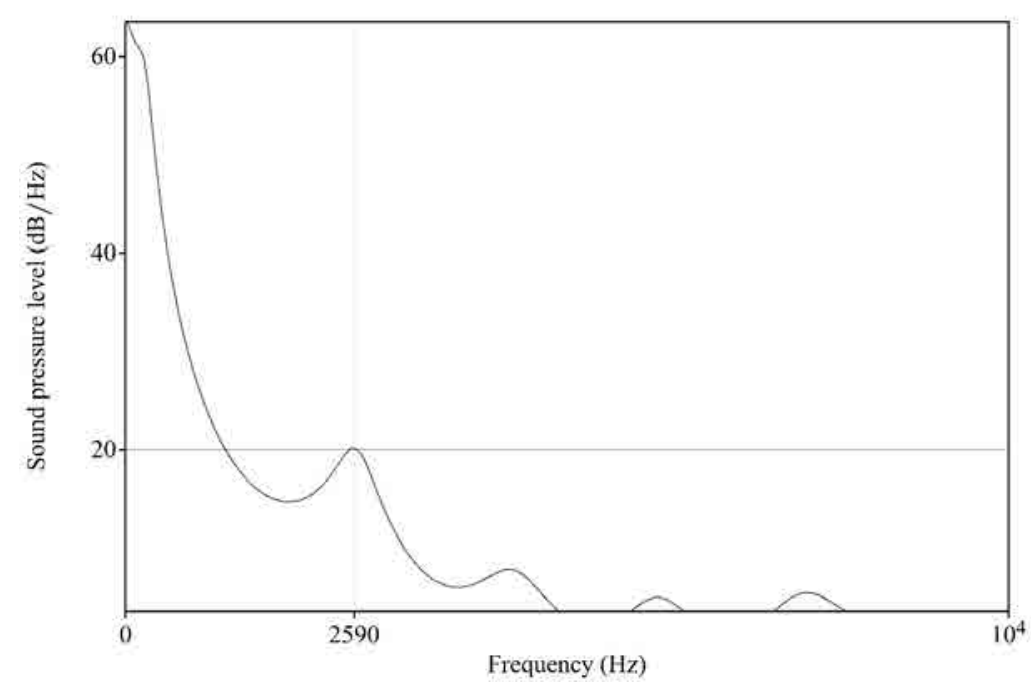

Nota. Se señala el pico más prominente de la consonante nasal bilabial sorda [m]-

Figura 9. Espectro LPC de la consonante nasal sorda [n]

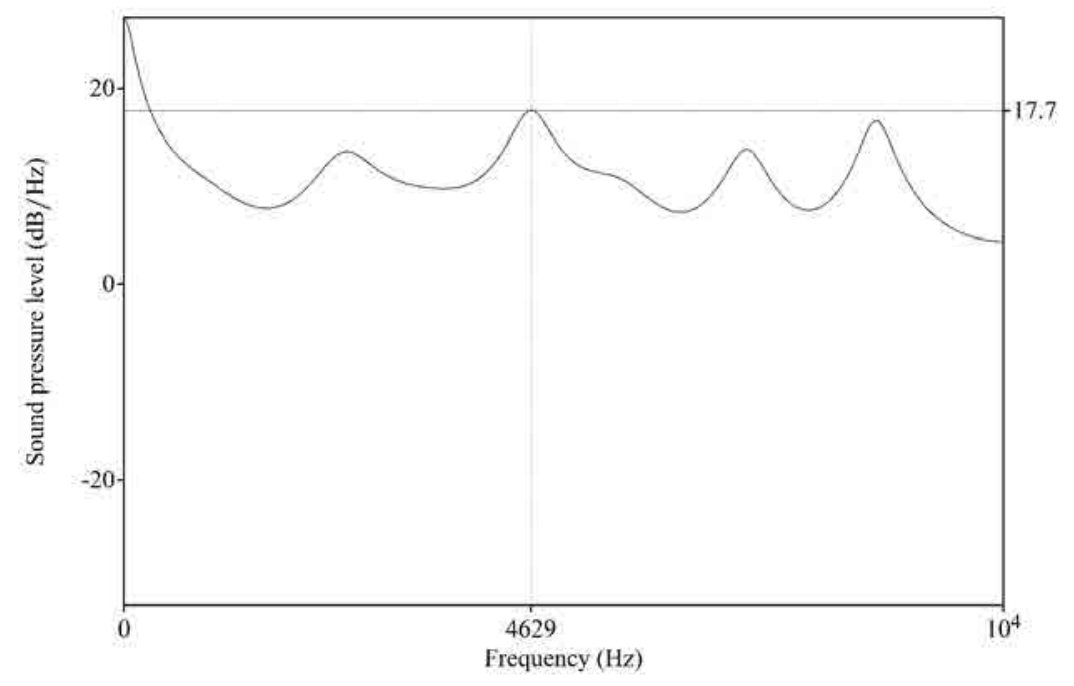

Nota. Se señala el pico más prominente de la consonante nasal alveolar sorda [n] ]. 
Un estudio fonético-acústico preliminar sobre las consonantes nasales sordas de la... Jhon Jimenez Peña

\subsection{Duración}

Dantsuji (1984) presenta también la duración de ambas porciones y total de las nasales sordas en el birmano. Se midieron las porciones de fricción nasal y nasal sonora de quince nasales bilabiales sordas y quince alveolares sordas. En la Tabla 3 y Tabla 4, presentamos lo resultados que corresponden a las producciones realizadas por el hablante.

Tabla 3. Promedio de la duración de las partes de la consonante nasal alveolar sorda $[\mathrm{m}]$

\begin{tabular}{l|l|l|l|l} 
& tokens & $\begin{array}{l}\text { Porción de } \\
\text { fricción }\end{array}$ & $\begin{array}{l}\text { Porción de } \\
\text { sonoridad }\end{array}$ & Total \\
\hline$\left[\mathrm{m}_{0}\right]$ & 15 & 0.107 & 0.032 & 0.139 \\
\hline
\end{tabular}

Nota. El tiempo está expresados en segundos

Tabla 4. Promedio de la duración de las partes de la consonante nasal alveolar sorda [n]

\begin{tabular}{l|l|l|l|l} 
& tokens & $\begin{array}{l}\text { Porción de } \\
\text { fricción }\end{array}$ & $\begin{array}{l}\text { Porción de } \\
\text { sonoridad }\end{array}$ & Total \\
\hline$\left[\mathrm{n}_{0}\right]$ & 15 & 0.104 & 0.045 & 0.149 \\
\hline
\end{tabular}

Nota. El tiempo está expresados en segundos

De las Tablas 3 y 4, se desprende que la duración de la porción de la fricción nasal es mayor que la porción de la parte de nasal sonora en ambas nasales en resígaro. Estos resultados obtenidos concuerdan con los de Dantsuji (1984) para el birmano.

\subsection{Clasificación de las nasales del resígaro a partir del análisis fonético-acústico}

En la sección del marco referencial de este artículo, se indicó que Bhaskararao y Ladefoged (1991) dividen en dos grupos a las nasales sordas de tres lenguas tibero-burmano. Por un lado, el grupo 1 está constituido por el birmano y mizo. Este 
Un estudio fonético-acústico preliminar sobre las consonantes nasales sordas de la...

Jhon Jimenez Peña

grupo se caracteriza por presentar la porción de fricción y porción de nasal sonora. El segundo grupo lo constituye el angami. La característica más resaltante que presenta este es que el flujo de aire nasal se extiende más generando aspiración. El resígaro no podría ser clasificado en este último grupo puesto que no presenta señales de aspiración, sino en el primer grupo ya que presenta marcadamente ambas porciones.

\section{Conclusiones}

a. Por medio del análisis a partir de espectrogramas, se detectó que las consonantes nasales sordas $\left[\mathrm{m}_{\mathrm{o}}\right]$ y $\left[\mathrm{n}_{\mathrm{o}}\right]$ del resígaro presentan dos características fundamentales: una porción de fricción y otra de sonoridad. La primera es ruido que se concentra a lo largo del segmento y la segunda es corresponde sonoridad con poca duración al final del segmento.

b. El análisis espectral LPC ha permitido observar la intensidad de la porción de fricción de las consonantes nasales sordas. La frecuencia de los picos de mayor intensidad $\left[\mathrm{m}_{\mathrm{o}}\right]$ y $\left[\mathrm{n}_{\mathrm{o}}\right]$ están distanciados significativamente de manera que esta podría ser uno de los parámetro que diferencia la una de la otra. No se pudo obtener la frecuencia del pico de mayor intensidad de $[\mathrm{n}]$ ya que no se identificaron adecuadamente en los datos.

c. En cuanto la duración, la parte de fricción nasal presenta mayor duración que la parte nasal sonora de las consonantes nasales sordas $\left[m_{0}\right]$ y $\left[n_{0}\right]$. Este aspecto concuerda con la afirmación que Dantsuji (1984) ofrece para el birmano.

d. Se propone, tentativamente, agrupar al resígaro en el mismo grupo del birmano y mizo (no filogenéticamente, claro está) por sus características acústicas semejantes ya descritas en el presente artículo.

e. Este artículo debe considerarse como un análisis preliminar sincrónico de las características acústicas de las consonantes nasales sordas del resígaro. Es necesario profundizar más sobre la naturaleza de esta clase de sonidos ya que es la única lengua del territorio peruano que presenta esta consonante. Además, es concerniente realizar estudios interlingüisticos con lenguas de la misma familia lingüística como el baniwa como también las siguientes lenguas de Sudamérica: koreguaje, warelema ayoreo y krenak con la finalidad de poder identificar similitudes y diferencias. Por último, el surgimiento de las nasales sordas del resígaro es incierto y necesita ser discutida en plano teórico y diacrónico. 
Un estudio fonético-acústico preliminar sobre las consonantes nasales sordas de la... Jhon Jimenez Peña

Agradecimientos

Este artículo es producto del trabajo de investigación elaborado para el curso «Análisis lingüístico I» (ciclo 2019-II) que se imparte en la maestría en lingüística de la Facultad de Letras y Ciencias Humanas de la Universidad Nacional Mayor de San Marcos. Agradezco infinitamente a Pablo Andrade Ocagane y Rosa Andrade Ocagane $(\dagger)$ por permitirme escuchar el resígaro a través de ellos en un periodo de tiempo muy corto, pero que atesoro grandemente. Gracias totales a María Giuliana Tito Quinteros por su amabilidad y gentileza en compartirme sus datos que ella recopiló del resígaro para complementar el estudio realizado. Además, un agradecimiento especial a Fernando Aaron Torres Castillo por la motivación para presentar este estudio.

\section{Financiamiento}

La investigación se realizó sin financiamiento por alguna entidad.

\section{Conflicto de intereses}

No se presenta conflicto de interés.

\section{Referencias Bibliográficas}

Allin, T. R. (1976). A grammar of Resígaro (Doctoral dissertation, The University of St Andrews).

Dantsuji, M. (1984). A Study on Voiceless Nasals in Burmese.

Dantsuji, M. (1986). Some acoustic observations on the distinction of place of articulation for voiceless nasals in Burmese.

Bhaskararao, P., \& Ladefoged, P. (1991). Two types of voiceless nasals. Journal of the International Phonetic Association, 21(2), 80-88.

Blankenship, Barbara, Peter Ladefoged, Peri Bhaskararao y Nichumeno Chase. 1993. Phonetic structures of Khonama Angami. Fieldwork studies of targeted languages (UCLA Working Papers in Phonetics) 84, 127-141.

Boersma, P., y Weenink, D. (2018). Praat: Doing Phonetics by Computer (Versión 5.4.17). Obtenido de http://www.fon.hum.uva.nl/praat/

(ILV). (2006). Pueblos del Perú. Lima: ILv.

Chirkova, K., Basset, P., \& Amelot, A. (2019). Voiceless nasal sounds in three TibetoBurman languages. Journal of the International Phonetic Association, 49(1), 1-32. doi:10.1017/Soo25100317000615

Chomsky, N. y Halle, M. (1979). Principios de fonología generativa. Fundamentos.

$310 \quad$ Lengua y Sociedad. Revista de Lingüística Teórica y Aplicada 
Un estudio fonético-acústico preliminar sobre las consonantes nasales sordas de la... Jhon Jimenez Peña

Elías-Ulloa, J. (2011). Una documentación acústica de la lengua shipibo-conibo (pano). (Con un bosquejo fonológico). Lima: PUCP.

Fagua, Doris. (2014). Género y sufijos clasificatorios de discretización en ocaina (witoto). Revista Brasileira de lingüística antropológica, 6 (1), 87-103

Harrington, J. (2010). Acoustic Phonetics. (W. J. Hardcastle, J. Laver, \& F. E. Gibbon, Edits.) The Handbook of Phonetic Sciences, 81-129.

Jimenez Peña, J. (2018). Los sonidos de la lengua arabela: un bosquejo fonológico. Lima: UNMSM.

Johnson, K. (2003). Acoustic and Auditory Phonetics (2 ed.). Cambrige: Blackwell Publishing.

Inventarios Fonológicos Suramericanos (sAphon v2.1.o)

Ladefoged, P. (2003). Phonetic data analysis. An introduction to fieldwork and instrumental techniques. Malden: Blackwell .

Ladefoged, P., \& Maddieson, I. (1996). The sounds of the world's languages (Vol. 1012). Oxford: Blackwell.

Ladefoged, P., \& Johnson, K. (2010). A Course in Phonetics (6 ed.). Boston: Wadsworth.

Maddieson, I. (1984). Patterns of sounds. Cambridge : Cambridge University Press. minedu. (2018). Documento nacional de lenguas originarias del Perú. Lima: MINEDU.

Minedu. (2017). Diagnóstico sociolingüístico de la lengua indígena resígaro. (Documento inédito)

Ohala, J. J., \& Ohala, M. (1993). The phonetics of nasal phonology: theorems and data. In Nasals, nasalization, and the velum (pp. 225-249). Academic Press.

Payne, D. L. (1985). The genetic classification of Resígaro. International Journal of American Linguistics, 51(2), 222-231.

sIL. Toolbox (versión 1.6.2)

SIL. Phonology Assistant (versión 3.5.4) 
Un estudio fonético-acústico preliminar sobre las consonantes nasales sordas de la... Jhon Jimenez Peña

\section{Trayectoria académica del autor}

Jhon Jimenez Peña es licenciado en Lingüística por la Universidad Nacional Mayor de San Marcos. Sus intereses están centrados en la fonética y fonología de las lenguas originarias del Perú, con especial atención a la lengua arabela. Ha sido consultor para el Ministerio de Educación para la elaboración de fonologías para los procesos de normalización de alfabetos del arabela, ocaina y taushiro. También, ha sido docente de los cursos de Fonología y Fonología Avanzada en el Curso Internacional de Lingüística, Traducción y Alfabetización (CILTA) del Instituto Lingüístico de Verano en los años 2018, 2019 y 2020 que se imparte en la Universidad Ricardo Palma. Además, ha sido expositor para el primer «CursoTaller de fonética forense» organizado por el CILA-UnMSM. Es miembro del grupo de investigación Dolenper: Documentación lingüística de lenguas amenazadas en el Perú (CILA-UnMSM). Actualmente, labora como perito lingüista forense en la Oficina de Peritajes del Ministerio Público-Fiscalía de la Nación y es miembro del Gabinete de Lingüística forense del CILA-UnMSM.

312 Lengua y Sociedad. Revista de Lingüística Teórica y Aplicada 